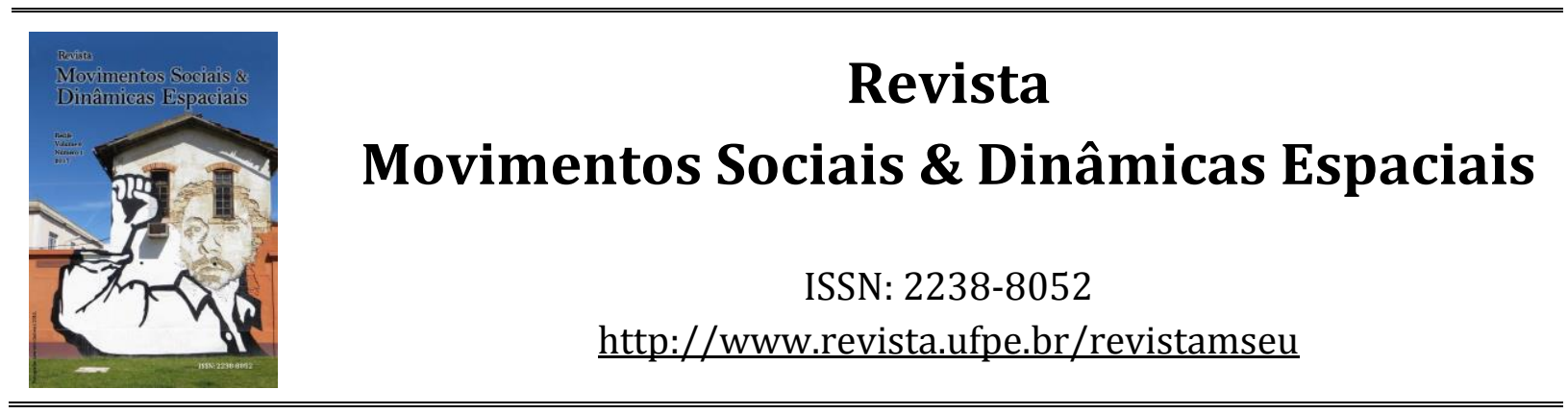

Artigo recebido em 14/05/2017 e aceito em 13/07/2017.

\title{
ACCUMULAZIONE E SPOLIAZIONE DELLA BIOSFERA IN SICILIA ORIENTALE - APPUNTI PER L'ELABORAZIONE DI UN MODELLO DI LETTURA DELLE CRISI SOCIO-ECOLOGICHE
}

\author{
ACUMULAÇÃO E ESPOLIAÇÃO DA BIOSFERA NA SICÍLIA ORIENTAL \\ APONTAMENTOS PARA A ELABORAÇÃO DE UM MODELO DE LEITURA DAS \\ CRISES SOCIO-ECOLÓGICAS
}

Salvo TORRE 1

\begin{abstract}
RIASSUNTO
Il caso della Sicilia Orientale può essere utilizzato per ragionare su un modello di lettura delle modalità con cui nell'ultimo quarantennio si sono verificate diverse crisi ecologiche e sul modo con cui si sono affermati molti processi di impoverimento ed erosione delle risorse. Il quadro che emerge è quello di un'area mediterranea in cui la crisi industriale degli anni Settanta del Novecento non ha prodotto un alleggerimento della pressione sull'ambiente locale, si sono solo modificate le modalità di sfruttamento. I principali processi che si sono realizzati, come la dismissione industriale, la crisi dell'agricoltura, la nascita dei nuovi poli commerciali e l'espansione urbana, hanno avuto uno stretto legame con le grandi trasformazioni globali e hanno partecipato alla produzione di una crisi ambientale. Il risultato semba essere un quadro in cui l'insieme delle attività umane è stato segnato dai processi di accumulazione per spoliazione e dall'estrazione di valore dalla biosfera.
\end{abstract}

Parole-chiave: Crisi ecologiche, accumulation by dispossession, ecologia politica, ambiente Mediterraneo.

\section{RESUMO}

O caso da Sicília Oriental pode servir de parâmetro para se pensar sobre um modelo de leitura das modalidades através das quais, nos últimos quarenta anos, foram verificadas diversas crises ecológicas bem como sobre o modo por meio do qual se consolidaram muitos processos de empobrecimento e erosão dos recursos. 0 quadro ora em análise refere-se a uma área mediterrânea na qual a crise industrial dos anos Setenta do século XX não conseguiu atenuar a pressão sobre o ambiente local, apenas se modificaram as modalidades da sua exploração. Os

1 Professor da Università degli Studi di Catania (UNICT)/FLACSO España. E-mail: s.torre@unict.it. 
principais processos realizados, tais como o fechamento das indústrias, a crise da agricultura, o nascimento dos novos polos comerciais e a expansão urbana, tiveram estreita ligação com as grandes transformações globais e participaram da produção de uma crise ambiental. 0 resultado parece conjuminar em um quadro no qual o conjunto das atividades humanas foi ressignificado pelos processos de acumulação visando à espoliação e pela extração de valores da biosfera.

Palavras-chave: Crise ecológica, Acumulation by dispossession, ecología política, ambiente Mediterrâneo.

\section{LO SCENARIO ATTUALE}

La Sicilia sta affrontando una complessa crisi socio-ecologica che rischia di compromettere per lungo tempo le possibilità di riorganizzazione della società e della biosfera locali. La crisi è il risultato diretto delle trasformazioni economiche e sociali degli ultimi decenni, dipende dalla destrutturazione progressiva delle relazioni tra le comunità locali e l'ambiente, ma anche dall'assenza di qualunque progetto di riorganizzazione del territorio. Negli ultimi decenni, si è realizzato un processo che ha ridefinito il funzionamento generale di tali relazioni, ma che ha colpito anche l'ormai precario funzionamento dei biomi locali.

Per comprendere il caso siciliano, bisogna considerare ovviamente la notevole profondità delle relazioni tra le comunità umane e la biosfera, perché la storia ambientale dell'area è chiaramente condizionata dalla forte presenza di quelle attività produttive di lungo periodo che hanno ridefinito tutta l'area mediterranea (HORDEN e PURCELL, 2000; BRAUDEL, 2008). L'intero territorio dell'isola è stato attraversato infatti da molte attività umane, che hanno costruito con le specie viventi un insieme di relazioni funzionali all'economia, come, ad esempio, le antiche forme di agricoltura o l'estrazione mineraria del Novecento. La notevole profondità storica delle relazioni comporta dunque la necessità di rileggere alcuni processi di lungo periodo insieme alle grandi novità e di ripensare alle permanenze storiche e culturali che si ritrovano ancora negli insediamenti umani. Sicuramente la successione di ondate di strutture sociali, che si sono affermate nel corso di almeno tre millenni, ha trasformato irreversibilmente l'area (FARINELLI, 2007); nel corso del tempo le varie comunità hanno costituito le loro relazioni, hanno ristrutturato l'ambiente locale e hanno operato interventi di modifica che condizionano ancora le possibilità di sviluppo. La fase attuale è però anche la diretta espressione della ristrutturazione avvenuta nell'ultimo quarantennio. Un'analisi generale del sistema locale dovrebbe partire quindi dalle modalità con cui durante la tarda modernità l'intera biosfera è stata trasformata in un deposito di valore, soprattutto nella fase di maggiore espansione dell'economia capitalistica, tenendo anche in considerazione le fasi storiche in cui la Sicilia era inserita nel sistema mondiale delle relazioni commerciali coloniali. Ripensando alla storia recente, si può però costruire una prima lettura delle 
modalità con cui i processi di ristrutturazione dell'economia a partire dagli anni Settanta del Novecento hanno ridefinito l'insieme delle relazioni ecologiche dell'isola.

A ridosso della crisi degli anni Settanta, si è avviato un processo che ha ormai già ridisegnato l'insieme della biosfera locale, anche perché, nei prossimi decenni, i risultati di ciò che è avvenuto rappresenteranno il principale problema ambientale locale. Ciò che è stato determinante, nell'ultimo quarantennio, è inoltre solo la declinazione locale di un mutamento che ha interessato l'intero pianeta e che si può sintetizzare analizzando due aspetti: l'estremizzazione dei processi di accumulazione per spoliazione e la progressiva dipendenza della biosfera dalla finanziarizzazione dell'economia. Osservando le modalità con cui si è determinata la crisi locale nell'area orientale dell'isola, si possono evidenziare inoltre alcuni elementi che possono essere collocati in uno schema utile alla definizione delle crisi socio-ecologiche recenti. Una storia ambientale ristretta esclusivamente agli ultimi quarant'anni è dunque una costruzione teorica che ruota intorno ai processi di accumulazione locali, definibili in termini molto precisi come azioni di spoliazione delle risorse, perché riferibili alla sottrazione di ricchezza e di capacità riproduttiva al sistema biologico locale.

Quella che è stata definita in vari modi come accumulation by dispossession (HARVEY, 2010; ARRIGHI, ASCHOFF e SCULLY, 2010; MOORE, 2011) non riguarda solo il suolo o la rendita fondiaria, ma tutto ciò che può essere considerato come appartenente alla categoria di bene comune. Anche nel caso della Sicilia Orientale, i beni comuni sono stati sottratti alla popolazione locale e l'equilibrio della biosfera è stato colpito dai processi di riconversione a valore o ad uso produttivo delle risorse. Nell'ultimo quarantennio, in corrispondenza con diverse fasi della crisi globale (AMIN, ARRIGHI, FRANK, WALLERSTEIN, 1982; FOSTER, 2011) il processo si è acuito, l'azione di spoliazione è diventata più diretta, ha colpito più duramente le risorse e le modalità di riproduzione. Ciò che il caso siciliano potrebbe dimostrare è dunque che il processo di spoliazione è una costante del funzionamento del sistema economico, non si realizza solo a sostegno dello sviluppo industriale, permane e si differenzia anche nelle fasi di dismissione degli impianti o di espansione del settore terziario.

I nodi di crisi ecologica presenti nell'area sono molti, la loro individuazione è probabilmene più semplice se si segue il processo storico che ha trasformato l'area e definito gli spazi di sottrazione delle risorse alle comunità e alla biosfera. Il processo di accumulazione per spoliazione è avvenuto infatti sia all'interno dello spazio urbano sia al di fuori, perché il valore si estrae primariamente dal suolo e dalla biosfera. Si è espresso però pienamente nella definizione dei margini, nella costruzione e nel mantenimento di aree povere nei contesti urbani e agricoli. La sottrazione delle risorse alla comunità corrisponde ovviamente all'impoverimento della popolazione locale, anche quando si realizza attraverso forme di inclusione nelle attività produttive. I processi di spoliazione sono anzi sostenuti dalle fasi espansive, ad esempio dall'aumento 
dell'occupazione o dalla costruzione di nuovi quartieri residenziali. I gruppi più deboli della popolazione subiscono però più direttamente le crisi ecologiche (MARTÍNEZ ALIER, 2004), sono i più colpiti dai danni ambientali e dalla riduzione di risorse, sono i primi a subire le conseguenze delle fasi di contrazione dell'economia, ma si ritrovano anche a partecipare alle attività a più alto tasso di inquinamento.

La fase attuale, ad esempio, si caratterizza particolarmente per i processi di esclusione a livello globale, il margine si riproduce e i contesti ambientali esplodono. Se si applica una lettura di tipo socio-ecologico, si possono evidenziare vari nodi del problema locale, la maggior parte dei quali dipende dalla declinazione locale di problemi ad ampio raggio che rispondono tutti allo stesso principio, come lo scarico di rifiuti tossici, il crollo della qualità della vita urbana, la desertificazione, il confinamento dei migranti. (Figura 1)

Figura1: Uso del suolo in Sicilia.



Fonte: Elaborazione su dati Corine Land Cover, 2012.

Un problema fondamentale, che emerge da questa storia, riguarda il fatto che tutto è avvenuto in stretta relazione con i processi globali, con i mutamenti del mercato mondiale e con la delocalizzazione della produzione. La spoliazione è sempre legata a due fattori: il primo è l'esistenza di un'economia del sistema-mondo, il secondo è la finanziarizzazione progressiva delle risorse ambientali. Le trasformazioni locali, per quanto legate a decisioni politiche e 
amministrative o all'azione di gruppi sociali, dipendono strettamente dalle trasformazioni che avvengono a livello generale, in questo caso nel sistema mediterraneo, oltre che in quello globale. Un momento di grande trasformazione del tessuto locale, ad esempio, corrisponde chiaramente agli anni della grande crescita dell'economia occidentale (HOBSBAWM, 1994; ARRIGHI, 1994), in cui si verifica anche la maggiore crescita economica italiana. Per la società dell'isola in quegli anni è il momento in cui si determina una forte emigrazione diretta verso l'Europa centrosettentrionale e il Nord Italia, ma anche quello in cui si chiude definitivamente la storia dei contadini meridionali. Il periodo successivo, in cui nell'area si realizza un esperimento fallimentare di creazione di un tessuto industriale, segue allo stesso modo i processi europei di delocalizzazione della produzione e quelli euromediterranei di conversione verso il terziario. Dagli anni Settanta, anche nella Sicilia Orientale si avverte una precisa corrispondenza tra declino economico, finanziarizzazione del sistema e saccheggio delle risorse. Tra il notevole numero di interventi che si differenziano sul territorio, si possono isolare quattro casi che sono molto utili a chiarire le modalità con cui si è realizzato il processo di spoliazione ai fini dell'accumulazione. Non si tratta degli unici spazi di azione di tali processi, però hanno contribuito a definire lo spazio sociale locale, l'uso del suolo, le relazioni ecologiche in genere: la fase finale di attività e la chiusura dei poli chimici siciliani, l'espansione urbana, la conversione dell'agricoltura e la costruzione dei sistemi della grande distribuzione commerciale. Nell'insieme si tratta di processi comuni a buona parte del pianeta, che si determinano in modo similare, soprattutto dopo la crisi, all'interno della globalizzazione, ma che possono rappresentare anche risposte generali del sistema. Rientrano inoltre tra quegli oggetti di analisi in cui è possibile notare in modo evidente l'applicazione dei modelli neoliberali di gestione delle risorse.

Il nodo centrale rimane l'accelerazione, con la crisi di metà anni Settanta, dei processi di globalizzazione e la progressiva applicazione di formule neoliberali di gestione delle risorse. Ciò che è avvenuto in Sicilia è il progressivo affermarsi del modello che adesso sta trascinando verso una serie di crisi ecologiche globali il pianeta, riproducendo processi di spoliazione di risorse e di esclusione di quote progressivamente più ampie di popolazione. Nel caso siciliano ovviamente permangono dinamiche che caratterizzano l'isola ancora come area periferica di un'economia più ricca e ciò ha consentito negli ultimi anni di mantenere ancora una qualità della vita superiore a quella delle aree più compromesse, inoltre la permanenza di reti sociali e una nuova fase migratoria hanno contenuto l'esplosione di nuove forme di povertà assoluta. La biosfera locale ha subìto però una lunga serie di danni che ne hanno modificato definitivamente gli equilibri e ne hanno compromesso le capacità riproduttive. (Figura 2) 
Figura 2: Aree maggiormente urbanizzate e aree industriali nella Sicilia Orientale
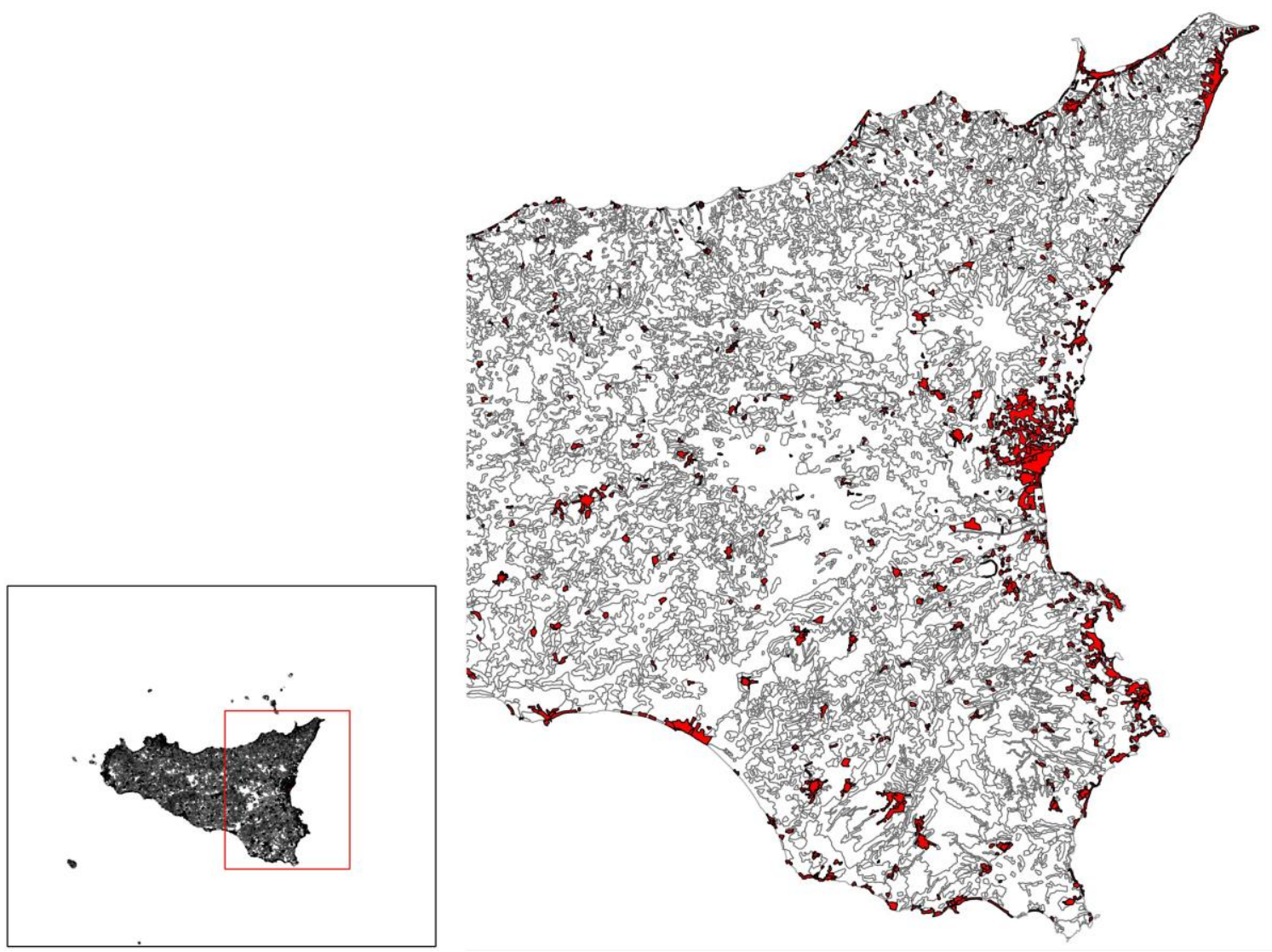

Fonte: Elab. Corine Land Cover 2012.

\section{IL DESERTO DELLA CHIMICA}

Il caso più eclatante di crisi ecologica locale è stato prodotto dalla costruzione e dalla dismissione del polo chimico di Augusta-Priolo-Melilli. Ciò che si è realizzato, tra la costruzione dell'impianto e la sua sostanziale chiusura, è stata la distruzione di tutto ciò che si poteva trovare prima. Adesso sembra inoltre impossibile trovare una soluzione, operare un recupero dell'area in tempi inferiori ad alcuni secoli. L'area del polo chimico della Sicilia Orientale è oggi una tra le più inquinate del pianeta (ADORNO, 2007; ADORNO e SERNERI, 2009). Il consumo di suolo non è una categoria adatta a descrivere ciò che è avvenuto, perché l'impatto della presenza degli impianti chimici si avverte su una scala molto ampia e ha modificato effettivamente l'intera storia ambientale dell'area. Durante gli anni di maggiore attività del polo chimico, la quantità di sostanze inquinanti diffusa nell'aria, nel suolo e nelle acque è stata tale che per valutare una seria bonifica dell'area bisognerà ancora attendere i risultati di diversi studi in atto. In molti casi infatti i dati rilevati rappresentano una novità assoluta e non si può fare riferimento a precedenti storici (RAMISTELLA et al., 1990). 
Il primo grande scompaginamento è derivato probabilmente già dalla progettazione dello sviluppo industriale locale. La storia della grande industria chimica in Sicilia inizia infatti subito dopo la Seconda Guerra Mondiale, i primi progetti di insedimento industriale sono già del 1949. In Italia, in quel periodo, inizia un ampio dibattito sulla necessità del paese di allinearsi alla grande produzione chimica mondiale e di riconsiderare lo sviluppo capitalistico delle aree meridionali del paese (SAITTA, 2010; PALIDDA, 2014). L'intero impianto del polo chimico, che avrebbe dovuto seguire un progetto generale per l'isola, viene realizzato secondo una visione tradizionale della localizzazione delle aree produttive e con un uso notevole degli investimenti pubblici. I poli chimici vengono quindi collocati lungo la costa per ridurre le spese e facilitare i trasporti (Figura 3). L'intero processo però è interno ad uno sviluppo europeo del settore e alla tipica programmazione degli insediamenti industriali nel Mediterraneo. La collocazione dei poli chimici ha seguito infatti, nell'Europa Meridionale, un processo di localizzazione ottocentesco per cui sono stati realizzati impianti dall'enorme impatto ecologico. Un tipo di produzione ad altissimo tasso di pericolosità ambientale viene cioè collocato in un'area periferica, povera, del paese, così come avvenuto in parte della storia coloniale o come avviene adesso, su scala planetaria, per tutte le forme di delocalizzazione delle produzioni ad alto rischio. L'idea dello sviluppo ad alto tasso di inquinamento rimane adesso un nodo da sciogliere per diverse aree mediterranee e pone spesso in modo drammatico di fronte a specifiche forme assunte negli ultimi anni dal conflitto tra capitale e lavoro (CHICCI, LEONARDI e LUCARELLI, 2016).

Figura 3: La localizzazione dei poli chimici siciliani.

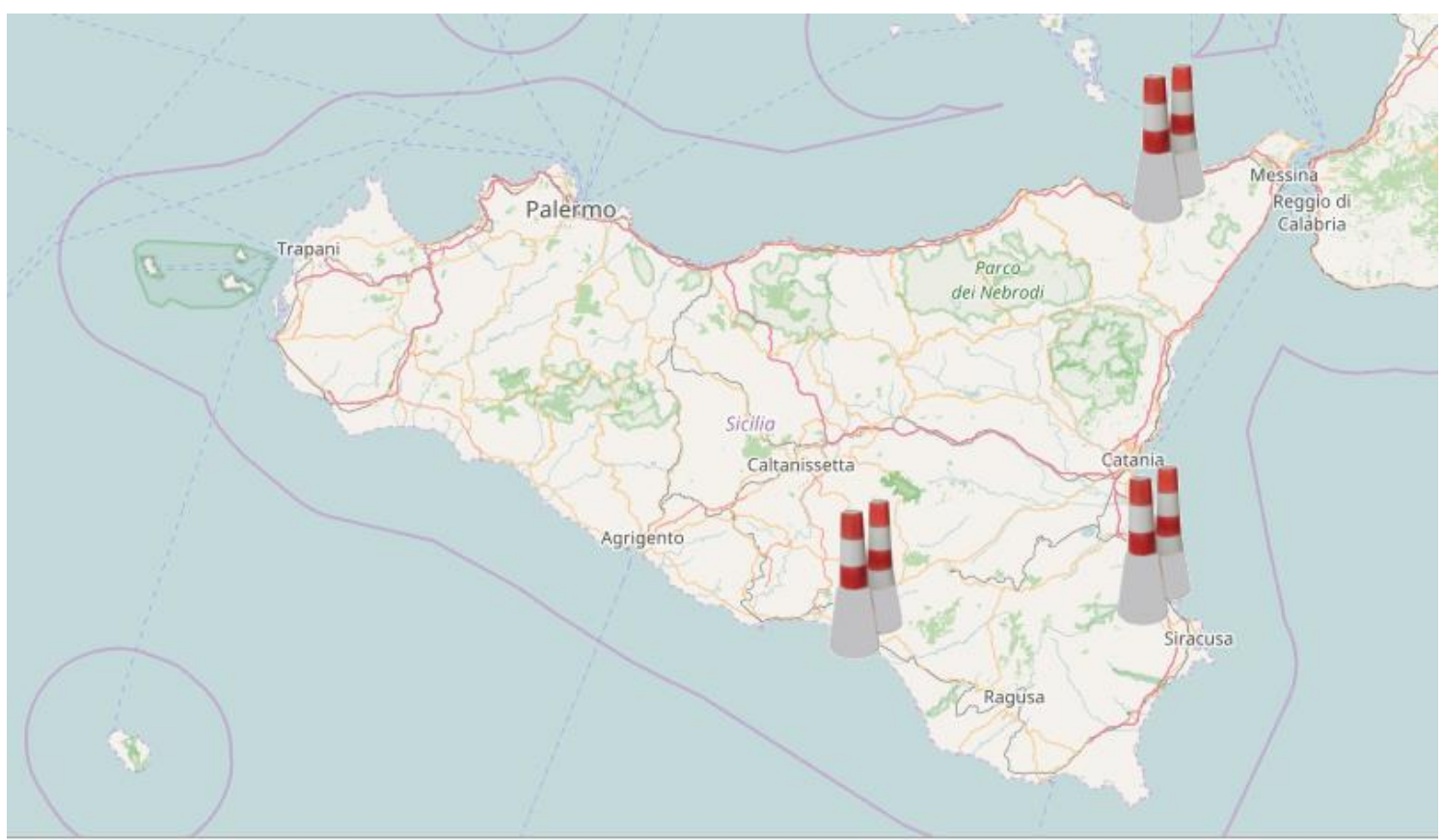


La storia della chimica siciliana finisce realmente alla fine degli anni Settanta del Novecento, la produzione entra in crisi in seguito allo shock petrolifero, nel decennio successivo galleggia cercando di utilizzare i fondi pubblici e dagli anni Novanta inizia il declino inesorabile. Meno di trent'anni hanno dunque modificato stabilmente l'area, producendo danni che si collocano in una sfera temporale di almeno sei secoli, perché adesso l'eredità ecologica della chimica siciliana si può calcolare solo sulla scala temporale dei mutamenti geologici (Tab.1). La chimica siciliana ha modificato stabilmente gli equilibri ambientali dell'isola, agendo in diversi casi come catalizzatore di problematiche mediterranee, in alcuni casi ha causato l'estinzione di specie locali, in altri ha determinato un mutamento sostanziale nei processi migratori e nelle dinamiche riproduttive di diverse specie. Ha inoltre condizionato l'espansione urbana di diversi poli (Catania, Siracusa, Gela), determinando lo spostamento di una notevole quantità di popolazione, così come la crescita e la crisi di diverse aree urbane, producendo anche danni sociali e sanitari di notevoli proporzioni. È chiaro anche che si è trattato degli anni della massima espansione locale dell'indutria dei combustibili fossili, in cui tutto il tessuto locale è stato convertito, dalle infrastrutture alle abitazioni, al modello della società del petrolio.

Ciò che è avvenuto nell'ultimo quarantennio è infine la dismissione dell'intero impianto, con una sottrazione progressiva dell'azione di controllo delle grandi imprese nazionali, sostituite da aziende globali negli ultimi anni, che hanno poi ridotto o trasferito la produzione. È stata una storia molto breve, in cui i processi di accumulazione hanno agito in modo lineare: espropriazione dei beni comuni e delle risorse statali, distruzione del sistema ambientale locale e abbandono dell'area, in seguito alla scoperta di luoghi in cui il costo della produzione e del lavoro erano più bassi. I poli chimici siciliani dimostrano sostanzialmente come quella che nel dibattito economico classico è definita caduta tendenziale del saggio di profitto sia stata risolta sempre aumentando la sottrazione di valore dalla biosfera. Per rispondere alla riduzione dei guadagni e all'aumento della competizione su scala globale, si è cercato di ridurre il costo del lavoro e di aumentare il danno ambientale, i due processi corrispondono sempre. Quando il sistema locale è entrato in crisi, anziché ridursi, il peso sulla biosfera locale si è solo differenziato, le attività umane hanno proseguito in varie forme il processo di estrazione di valore dalla biosfera. 
Tab.1 Rilevazioni inquinamento nell'area del polo chimico di Augusta-Priolo-Melilli. Nella colonna di destra il numero di volte in cui il materiale inquinante supera le soglie stabilite dall'OMS

\begin{tabular}{|c|c|c|}
\hline & Tipologia di inquinante & Superamento del valore limite \\
\hline \multicolumn{3}{|l|}{ Inquinamento del suolo } \\
\hline & $\begin{array}{c}\text { Metalli pesanti (arsenico, cromo, } \\
\text { mercurio) }\end{array}$ & 1000 \\
\hline & Idrocarburi & 300 \\
\hline & Composti aromatici (benzene) & 500 \\
\hline & IPA & 28 \\
\hline & Composti alifatici clorurati & 200 \\
\hline & Diossine & 20 \\
\hline \multicolumn{3}{|l|}{ Inquinamento della falda } \\
\hline & Arsenico & 130 \\
\hline & Mercurio & 50 \\
\hline & Benzene & 200000 \\
\hline & Toluene & 1600 \\
\hline & Cloruro di vinile & 24000 \\
\hline & Tricloroetilene & 2000 \\
\hline & Tetracloroetilene & 2500 \\
\hline & Esaclorobutadiene & 440000 \\
\hline & Tetracloroetano & 7000 \\
\hline & Dibromoclorometano & 130 \\
\hline & Clorobenzeni & 30000 \\
\hline & Idrocarburi totali & 800 \\
\hline
\end{tabular}

\section{IL SACCHEGGIO URBANO}

Negli stessi anni in cui si è consumata la crisi dell'industria chimica locale, si è realizzata la fase finale dell'esperienza di espansione urbana incontrollata che ha caratterizzato le città siciliane. Anche in questo caso si è trattato in parte della declinazione locale dei processi di crisi dell'edilizia italiana, in parte del mutamento delle città euromediterranee.

Quello che nel dibattito politico locale è stato definito come un saccheggio delle risorse urbane, si è realizzato con la sottrazione di spazi fisici, con un tasso di consumo di suolo eccezionale, considerata la ridotta dimensione delle città, ma anche attraverso la spoliazione di risorse storiche, come la distruzione di quartieri antichi o opere architettoniche. In generale, si è realizzata una sottrazione costante del diritto alla città (LEFEBVRE, 1972), costruita anche 
attraverso spostamenti di popolazione storica ed esclusione dai contesti di vita urbana degli abitanti di nuova immigrazione. Tra gli anni Cinquanta e Settanta del Novecento si tratta soprattutto di popolazione che si trasferisce dall'entroterra dell'isola e va ad ingrandire le aree urbane di Catania, Messina e Siracusa (Figura4). Dagli anni Settanta la costruzione di nuove aree assume quasi esclusivamente l'aspetto della speculazione ed è scollegata dall'incremento della popolazione o dalle esigenze reali di pianificazione. L'espansione urbana post crisi anni Settanta è meno programmata, ma ugualmente dannosa. L'idea che sostiene gli investimenti continua ad essere quella della costruzione delle città satellite e dei nuovi spazi residenziali, così in quegli anni i processi di accumulazione urbana cercano di compensare la crisi industriale e si inaspriscono, perché devono sostenere da soli la finanziarizzazione locale e producono una diffusione incontrollata delle aree urbane (DATO, 1984). Due esempi sono la sostanziale urbanizzazione della costa lungo tutto il tratto che congiunge Catania con Messina e il forte tasso di crescita delle aree periurbane.

Figura 4: Espansione area urbana catanese.

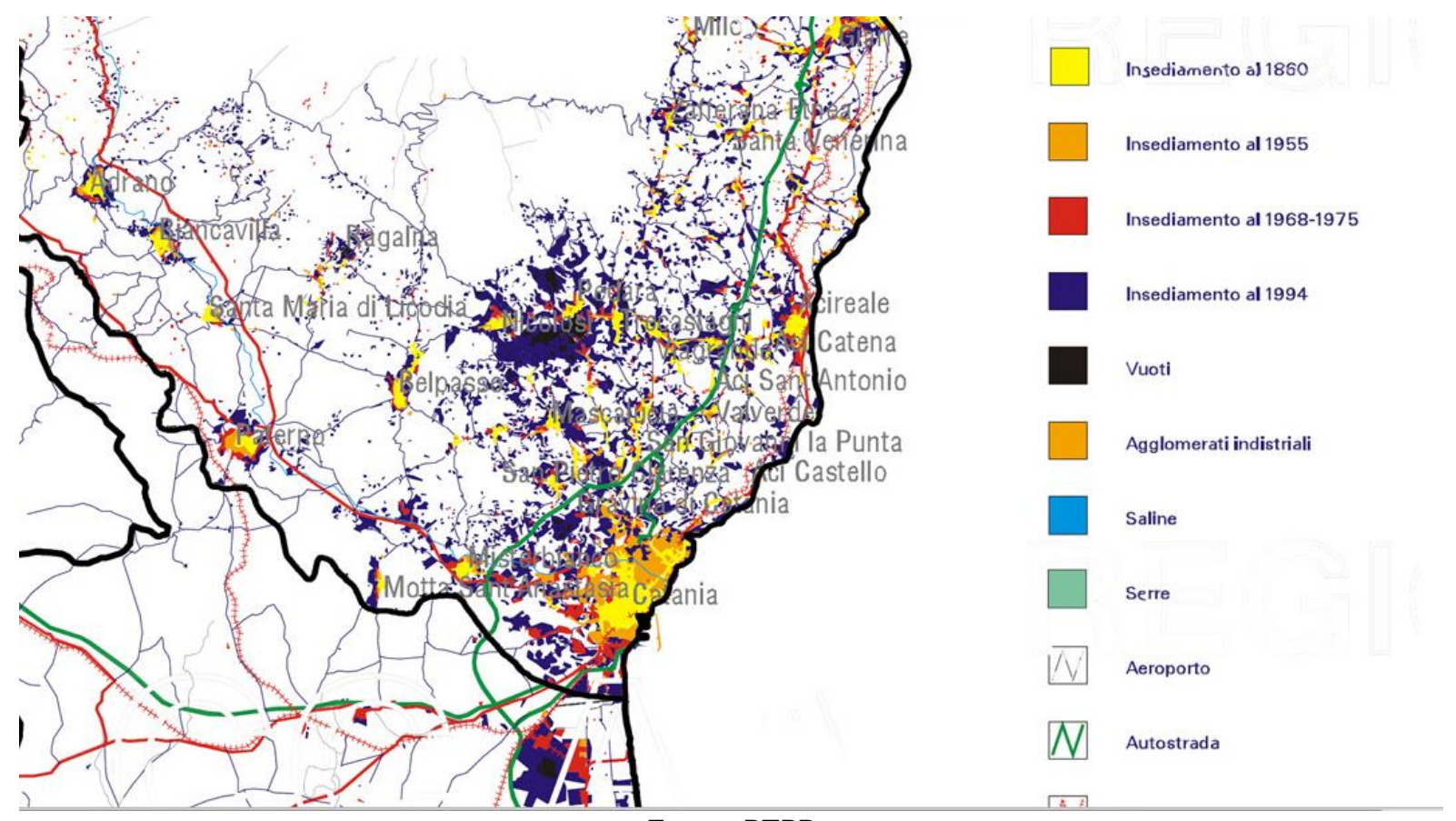

Fonte: PTPR.

La crisi ecologica corrisponde anche alla destrutturazione della socialità urbana e della cultura, la storia delle città viene decontestualizzata e le forme di espressione culturale vengono incanalate verso spazi ristretti che possono essere capitalizzati. Negli stessi anni si afferma un sistema di potere capillare frutto della compresenza del controllo del territorio da parte delle grandi organizzazioni criminali e del forte consenso politico ai partiti di governo. Si tratta di un sistema che coinvolge tutti gli aspetti della vita degli abitanti e che facilita la nascita di grandi 
processi di accumulazione, realizzati attraverso forme estreme di sfruttamento del territorio. Il settore dello smaltimento dei rifiuti, i settori di base dell'approvvigionamento dell'ediliza, la grande distribuzione commerciale, la costruzione di grandi opere, diventano in tutta l'area il luogo di grandi disastri ecologici prodotti dalle organizzazioni criminali. I risultati di quel processo sono oggi al centro di diversi nodi problematici ed anche di piccoli conflitti ambientali. Il sistema dello smaltimento dei rifiuti, ad esempio, ha prodotto diversi casi di conflittualità e pone adesso enormi problemi ambientali; in diversi casi la popolazione locale ha chiesto la chiusura e il trasferimento delle discariche a causa degli enormi rischi per la salute.

Dagli anni Settanta si realizza un modello di espansione periferica che è disastroso per l'ambiente locale, le città si allargano a dismisura, aumenta il consumo di suolo e le problematiche ambientali diventano diffuse. Il saccheggio ha colpito anche il contesto limitrofo alle aree urbane, non solo per l'espansione delle città, ma anche per la capacità di scaricare sui margini dello sviluppo i principali problemi, come lo smaltimento dei rifiuti tossici o la localizzazione delle attività pericolose. La pressione urbana colpisce, ad esempio, con queste due modalità le aree dei parchi naturali (Figura 5) in cui si collocano anche enormi problemi di dissesto idrogeologico, soprattutto nell'area del messinese.

Figura 5: Aree protette.

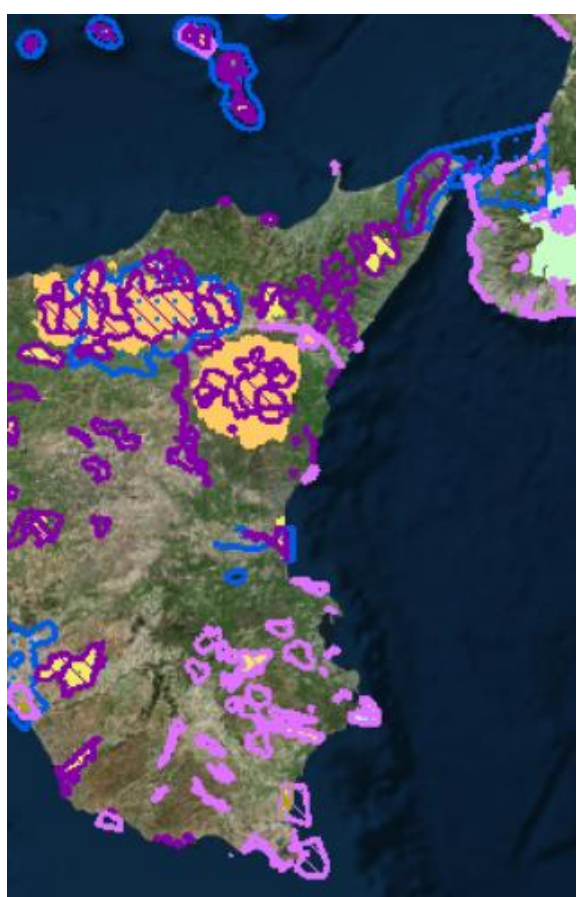

Fonte: Progetto Natura, Ministero dell'Ambiente.

L'espansione urbana è stata il luogo della principale fonte di accumulazione dell'isola, ha sostenuto l'economia locale, ha rappresentato il volano per l'occupazione della popolazione a 
basso reddito e ha modificato radicalmente gli assetti di vita della popolazione locale. Il risultato principale del processo, la grande accumulazione finanziaria, è stata l'impresa principale dell'area per diversi decenni. Solo gli affari delle grandi organizzazioni criminali hanno potuto sostenere il ritmo di crescita dell'edilizia locale, con cui infatti sono entrati spesso in relazione, e successivamente la nuova grande conversione al sistema della grande distribuzione commerciale.

Negli anni Novanta una serie di leggi per il recupero dei centri storici ha sostenuto un tentativo di gentrification che si è realizzato solo in parte, sostenuto soprattutto dall'economia turistica e del divertimento. Alcune aree dei centri storici, nelle tre città principali, sono state recuperate e convertite, la popolazione storica è stata progressivamente sostituita, soprattutto in funzione di un forte aumento della rendita fondiaria. L'industria delle costruzioni subisce una flessione evidente e traina l'espansione della crisi, ma l'attività si riduce, aumentando l'impatto per diffusione non per densità. L'andamento della crisi a partire dal 2008 ha contribuito alla riduzione delle attività di nuova costruzione, in Sicilia ad esempio negli ultimi anni le nuove edificazioni si sono mantenute sempre al di sotto dei 12.000 edifici annui nell'intera regione (ANCE, 2016). I quartieri delle nuove periferie degli anni Settanta e Ottanta pongono oggi notevoli problemi agli abitanti, rappresentano realisticamente le principali linee di esclusione dallo spazio urbano e non garantiscono molte prospettive di soluzione delle problematiche sociali. Ovviamente, la povertà urbana ha un elevato prezzo ecologico, sostiene economie devastanti dal punto di vista produttivo e alimenta un uso distorto dello spazio urbano.

Anche i processi di mutamento urbano sono stati sostenuti dall'accumulation by dispossession, perché sostanzialmente hanno sottratto le città alla loro popolazione, i beni comuni agli abitanti e distrutto la biosfera locale. Le città siciliane non assumono solo la fisionomia delle città del consumo (BECCHI, 2007), si espandono in modo incontrollato, aumentando esponenzialmente l'impatto dei nuovi edifici. Consumano suolo e distruggono biosfera, ampliando progressivamente il loro raggio di azione e distribuiscono in modo incontrollato i rifiuti provenienti dalla produzione locale e dal mercato globale.

\section{ULTIMA, L'AGRICOLTURA}

Nell'ultimo quarantennio si è consumata anche la definitiva crisi dell'agricoltura dell'isola, che oggi è diventata un settore sostanzialmente marginale. Il numero delle imprese è crollato e la produzione locale si è differenziata seguendo i ritmi dell'agricoltura Sud europea. Alcuni prodotti tradizionali sono stati sostituiti sul mercato europeo dai concorrenti.

L'agricoltura siciliana è oggi il luogo della presenza della fascia più debole delle migrazioni, soprattutto nelle aree più difficili della produzione delle serre, gestita in genere con un estremo 
sfruttamento del lavoro (AVALLONE, 2017) e in condizioni molto pericolose per la salute dei lavoratori e per la presenza di un grande quantitativo di prodotti chimici inquinanti. La produzione nelle serre ha un enorme impatto ambientale, paragonabile forse solo a quello dello smaltimento dei rifiuti. Così come l'edilizia era stata il luogo della manodopera sottopogata e più a rischio, ciò che resta dell'agricoltura dell'area è sostanzialmente il livello più basso del mercato del lavoro locale. Nel caso dell'area analizzata inoltre è finita anche l'esperienza di una delle maggiori area di produzione agrumicola del paese.

Un fattore importante di causa della crisi è stata l'applicazione della regolamentazione europea della produzione agricola, che ha imposto una suddivisione sostanzialmente legata al mercato globale dei prodotti e alla finanziarizzazione del sistema, non alla salvaguardia della biodiversità o delle produzioni tipiche. Seguendo lo schema di ciò che è avvenuto nell'Europa meridionale, diventa chiaro come l'agricoltura sia una delle aree di azione fondamentali per la globalizzazione neoliberale (MOORE, 2015); la redistribuzione delle aree di produzione e la destrutturazione dei vecchi mercati corrispondono infatti alla scomparsa di molti prodotti e soprattutto al contrasto all'autosufficienza alimentare. È chiaro anche che il regime agro-ecologico emerso subito dopo la crisi degli anni Settanta si è esaurito, ma la maggior parte delle aree dell'Europa meridionale, che prima mantenevano ancora margini di autosufficienza, sono diventate consumatrici di prodotti globali ed esportatrici di monocolture. In sé, non si tratta di un fenomeno del tutto nuovo nella storia dell'agricoltura capitalistica. Bisogna però tenere in considerazione come l'impatto delle nuove forme di commercio e distribuzione abbia condizionato in modo determinante la produzione locale e ridefinito la biosfera. Le tipologie di prodotti sono mutate e i prezzi imposti dalla grande distribuzione dei beni agricoli hanno ridotto la competitività delle merci locali. La questione centrale è dunque che l'intera isola oggi non è autosufficiente, consuma soprattutto prodotti che provengono da altre aree, la maggioranza dei quali è il frutto di forme di agricoltura ad elevatissimo impatto ecologico. Inoltre ciò che è sopravvissuto rappresenta ormai una tipologia di attività ad elevato tasso di inquinamento. Le comunità locali non possiedono oggi una reale sovranità alimentare, dipendono totalmente dal mercato globale, nonostante esistano ancora forme di resistenza nei mercati locali da parte di gruppi di produttori tradizionali e di nuovi gruppi che si oppongono alla spoliazione definitiva proponendo nuove forme di organizzazione della produzione e nuove forme di difesa della biodiversità.

La grande distribuzione si è sostituita all'industria come settore economico prevalente, in tutto il paese, ma nel caso siciliano è l'unico settore che rimane in crescita come numero di occupati e per apertura di nuovi poli. L'intera area è inquadrabile come uno spazio periferico di consumo, non come un centro produttivo. La costruzione dei nuovi poli commerciali ha seguito in tutta l'area lo schema dell'espansione territoriale, non ha cioè occupato gli spazi della dismissione, 
ma contribuisce ad allargare gli spazi urbani e sostiene interventi infrastrutturali, come strade e centri di interscambio, che modificano radicalmente il territorio (Nella zona delle tre aree urbane di Catania, Messina e Siracusa, al momento sorgono 27 aree definibili come centri commerciali, con la presenza delle tipiche gallerie commerciali, infrastrutture stradali e impianti di gestione dei magazzini per le merci, il numero però diventa più elevato se si considerano anche alcuni singoli impianti di grandi catene globali). Il costo ecologico dei nuovi spazi commerciali si è rivelato enorme ed oggi è uno tra gli elementi che definiscono più precisamente le relazioni socioecologiche; si tratta infatti di nodi in cui si sommano i vari modelli di sfruttamento, dal lavoro all'uso del suolo, e il cui impatto è enorme.

\section{COSA DEFINISCE LA CRISI LOCALE?}

Se si prende in considerazione la teoria del metabolismo rurale di Toledo (2008), cioè la sua teoria dell'appropriazione economico-ecologica della natura, bisogna comunque ammettere che la fase più recente della crisi economica ha reso evidente che i processi di appropriazione sono diventati più estremi. Non si tratta più solo del funzionamento tradizionale dell'economia capitalistica. La ricerca di risorse da convertire direttamente in valore, specificamente l'accelerazione del processo di finanziarizzazione ha ridotto la natura ad un deposito di valore e ha reso i beni comuni una persistenza di una struttura sociale da superare. L'aggressione condotta su vasta scala nel caso siciliano si è realizzata con un gran numero di modalità diverse, si è realizzata come sottrazione pressoché definitiva, su una scala temporale umana, di diversi territori all'uso sociale e alla biosfera - come nel caso dei poli chimici e delle discariche -, così come si è espressa nelle forme della sottrazione dei beni comuni alle comunità locali da parte di grandi aziende private - attraverso forme come l'espropriazione dell'accesso all'acqua -. Oggi è in atto una profonda crisi socio-ecologica, che si esprimerà in forme esplosive tra alcuni anni, ma che si può definire già adesso in modo complesso come somma dei processi che convertono in valore la biosfera e tendono ad utilizzare tutto, anche gli esseri umani, proprio in funzione della creazione di valore. La presenza di forme di sovraregolazione finanziaria si avverte pienamente nel contesto locale, attraverso l'imposizione ai produttori agricoli dei prezzi di vendita, l'andamento dei valori fondiari e la conversione finanziaria della rendita fondiaria urbana, la pressione del debito sugli enti locali. Dal punto di vista dell'analisi ambientale, su tutto il territorio sono presenti nodi di estrema precarietà, tutti caratterizzati ovviamente dall'evoluzione degli ultimi anni, dalla assoluta mancanza di intervento o dalla speculazione. I margini di tutte e tre le città sono costellati da aree dismesse, si intravedono ancora le permanenze delle strutture industriali dell'inizio dello scorso secolo, a cui si sono sommate le aree abbandonate dalle varie ondate di sviluppo. Negli ultimi anni 
sono nate alcune nuove aree vuote frutto della dismissione della fase di grande distribuzione commerciale dei tardi anni Ottanta del Novecento, entrata in crisi da una decina di anni. Una questione evidente è che con la contrazione dell'attività industriale non si è esaurito il processo di destrutturazione delle relazioni locali né si è ridotta la spoliazione delle risorse. Le aree urbane hanno continuato a crescere secondo uno schema riferibile all'accumulazione flessibile, ma in cui semplicemente la speculazione viene spostata in nuove aree senza riconvertire le vecchie. La città si allarga senza che avvenga alcuna forma di recupero delle zone abbandonate, così i nuovi parchi commerciali vengono realizzati in nuove aree e si candidano a diventare le nuove aree dismesse della prossima crisi di accumulazione locale. La dinamica del consumo estromette la merce dal sistema, perché la finalità del mercato è la distruzione della merce, cioè la dissipazione delle risorse per il sostegno a nuove forme di consumo. È evidente che la biosfera è all'interno della catena di consumo.

Ciò che è avvenuto in Sicilia è il risultato di un processo di lungo periodo, in cui la conversione delle comunità locali è avvenuta progressivamente, con alcune accelerazioni in corrispondenza della globalizzazione e dell'affermazione del modello neoliberale italiano. La storia dell'industria chimica finisce insieme alla congiuntura globale, la produzione agricola entra in crisi con la ristrutturazione della produzione europea e con la finanziarizzazione dell'agricoltura, l'edilizia finisce con l'esplosione della bolla speculativa globale e con il mutamento della struttura generale della popolazione, tutti i settori risentono della fine del modello di intervento statale novecentesco. I processi corrispondono inoltre alla radicalizzazione dell'azione di controllo e alla scomparsa della mediazione politica, sostituita ormai dalle forme di contrattazione neoliberale. I processi economici locali hanno progressivamente eroso le riserve ecologiche con modalità differenti, dalla distruzione prodotta dai poli chimici e dalle periferie industriali all'erosione del suolo agricolo, dalla desertificazione dell'interno all'erosione delle coste. (Figura 6) Sono tutti processi locali che si confronteranno con quelli planetari come il global warming già dalla fine di questo decennio. La modalità con cui ciò è avvenuto è stata propriamente l'accumulazione per spoliazione, cioè la costruzione di valore dalla sottrazione al territorio e alle comunità locali. Il modo in cui è avvenuto è anche il modo in cui il sistema si è mantenuto dopo la crisi degli anni Settanta e corrisponde alle modalità con cui il sistema globale sta sopravvivendo. I prossimi anni presenteranno probabilmente nuovi scenari di conflitto ambientale, le uniche forme che possono a questo punto ridisegnare le modalità di funzionamento delle comunità locali, anche nell'area mediterranea. 
Figura 6: Le aree a rischio desertificazione in Sicilia.

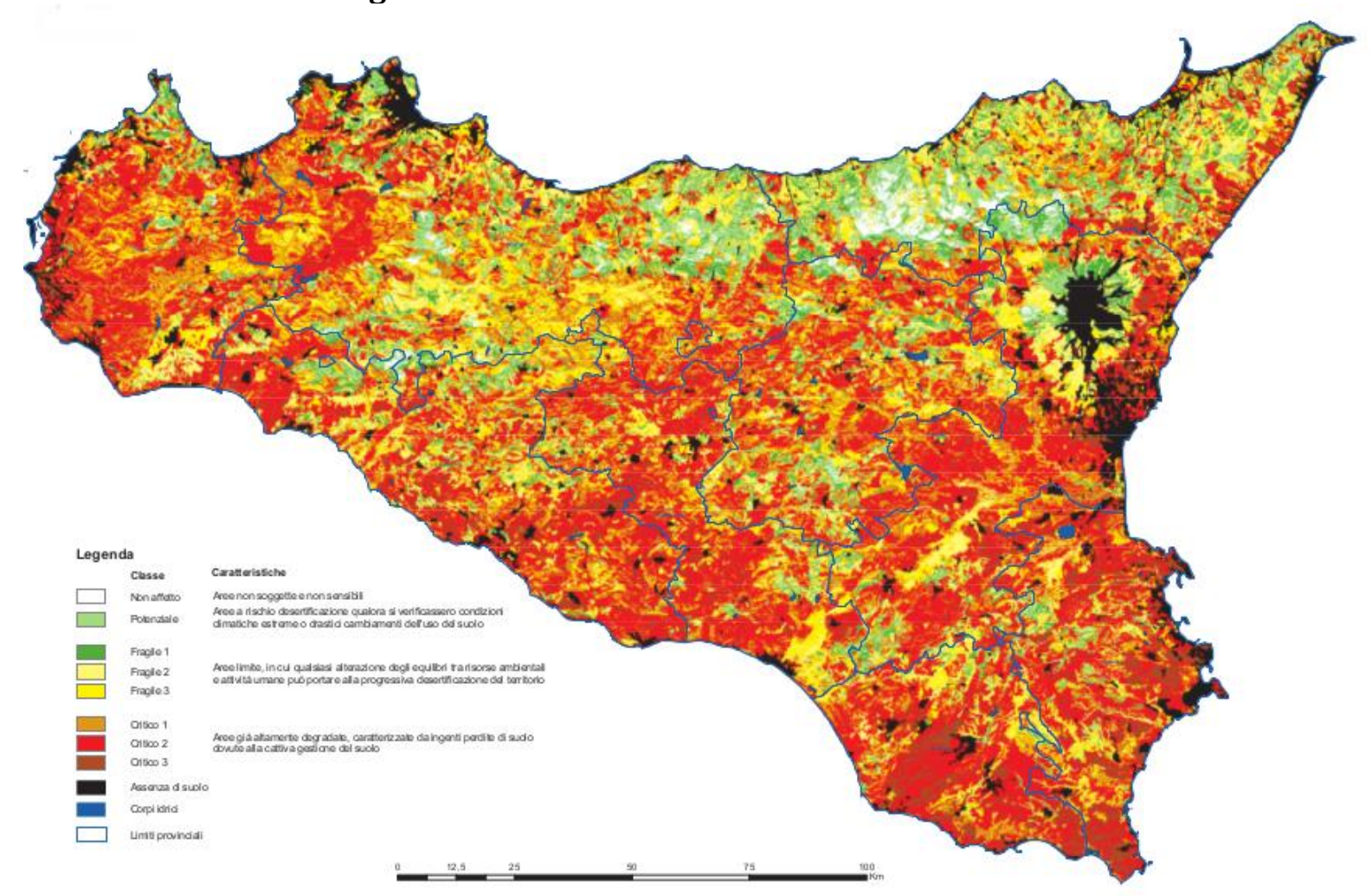

Fonte: F. Calvi, P. Catena, R. Cibella, A. Cirasa, F. Dolce, A. Drago, V. Ferraro, D. Galvano, A. Ganci, F. Gendusa, R. Giordano, Granata, F. Guaitoli, A. Lo Bello, M. G. Matranga, M.T. Noto, M. Perricone, Environmentally Sensitive Areas to Desertification, ESAs, Regione Siciliana.

\section{REFERÊNCIAS}

ADORNO, Salvatore, NERI SERNERI Simone, eds. Industria, ambiente e territorio: per una storia ambientale delle aree industriali in Italia. Bologna: Il Mulino, 2009.

ADORNO, Salvatore. L'inquinamento dell'aria e dell'acqua nel polo petrolchimico di Augusta - Siracusa nella seconda metà degli anni Settanta. Reti, controlli e indagini ambientali. I Frutti di Demetra, 15: 43-57, 2007.

A.N.C.E., Rapporto congiunturale sull'industria delle costruzioni in Sicilia. Roma: Associazione Nazionale Costruttori Edili, 2016

AMIN, Samir, ARRIGHI, Giovanni, GUNDER FRANK, André, WALLERSTEIN, Immanuel. Dynamics of Global Crisis. New York: Monthly Review Press, 1982.
ARRIGHI, Giovanni. The Long Twentieth Century: Money, Power and the Origins of Our Times. New York: Verso, 1994.

ARRIGHI, Giovanni, ASCHOFF, Nicole e SCULLY, Ben. Accumulation by Dispossession and Its Limits: The Southern Africa Paradigm Revisited. Studies in Comparative International Development, 45: 410-438, 2010.

AVALLONE, Gennaro. Sfruttamento $e$ resistenze. Migrazioni e agricoltura in Europa, Italia, Piana del Sele. Verona: Ombre Corte, 2017.

BARCA, Stefania e LEONARDI, Emanuele. Working-class communities and ecology. Reframing environmental justice around the Ilva steel plant in Taranto, Apulia (Italy), in M. Shaw, M. Mayo (eds.), Class, Inequality and 
Community Development. Bristol (UK) and Chicago (USA): Policy Press/Chicago University Press, 59-76, 2016.

BECCHI, Ada. Le città del consumo oggi. Scienze Regionali, 3: 113-136, 2007.

BRAUDEL, Fernand. Il Mediterraneo, Lo spazio, La Storia, gli uomini le tradizioni. Milano: Bompiani, 2008.

CHICCHI, Federico, LEONARDI, Emanuele e LUCARELLI, Stefano. Logiche dello sfruttamento. Verona: Ombre Corte, 2016.

DATO, Giuseppe. Urbanistica e città meridionale. Catania: Culc, 1984.

FARINELLI, Franco. Il Mediterraneo, la differenza, il differimento. Idee, 65:47-58, 2007.

FOSTER, John Bellamy. The Ecological Rift: Capitalism's War on the Earth. New York: Monthly Review Press, 2011.

HARVEY, David. The 'New' Imperialism: accumulation by dispossession. Socialist Register, 40: 63-87, 2004.

HOBSBAMW, Eric. The Age of Extremes: The Short Twentieth Century, 1914-1991. New York: Pantheon Books-Random House.

HORDEN, Peregrine e PURCELL, Nicholas. The Corrupting Sea. A Study of Mediterranean History. London: Blackwell, 2000.

LEFEBVRE, Henri. Le Droit à la ville suivi de Espace et politique. Paris: Anthropos, 1972.
MARTÍNEZ ALIER, Joan. El ecologismo de los pobres. Conflictos ambientales y lenguajes de valoración. Barçelona: Editorial Icaria, 2004.

MOORE, Jason W. Ecology, Capital, and the Nature of Our Times: Accumulation \& Crisis in the Capitalist World-Ecology. Journal of World-System Research, 17:107-146, 2011.

MOORE, Jason W. Capitalism in the Web of Life: Ecology and the Accumulation of Capital. New York: Verso, 2015.

PALIDDA, Salvatore, ed. Governance of Security and Ignored Insecurities in Contemporary Europe. London: Routledge, 2016.

RAMISTELLA, E. M., Bellia, M., Di Mare, S., Rotiroti, G., \& Duscio, D. Inquinamento ambientale di origine industriale e mortalità per tumore. Revisione della situazione di Augusta e Priolo. Bollettino dell'Accademia Gioenia di Scienze Naturali, 23(336): 43762, 1990.

SAITTA, Pietro. Il petrolio e la paura. Popolazioni, spazio e altra economia nelle aree a rischio siciliane. Roma: Aracne, 2010.

TOLEDO, Victor. Metabolismos rurales: hacia una teoría económico-ecológica de la apropiación de la naturaleza. Revista Iberoamericana de Economía Ecológica, 7: 126, 2008. 